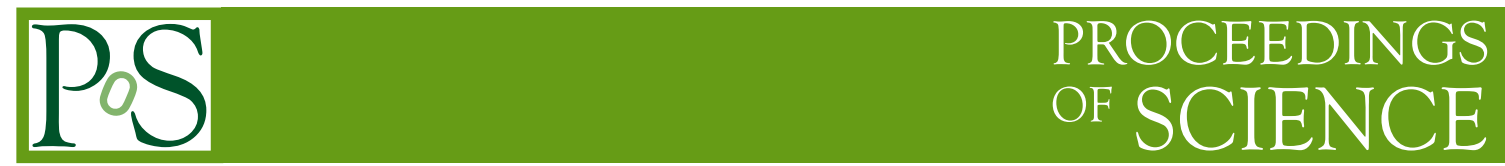

\title{
Associated Top Production at the LHC
}

\author{
Bárbara Álvarez González on behalf of the CMS and ATLAS Collaborations \\ Universidad de Oviedo, Instituto Universitario de Ciencias y Tecnologías Espaciales de Asturias \\ (ICTEA), Spain \\ E-mail: balvarez@cern.ch
}

Several new and exciting results on the associated top quark production at the LHC are presented. Data collected with the CMS and ATLAS experiments in proton-proton collisions at a centre-ofmass energies of $13 \mathrm{TeV}$ is used and the results are compared to the standard model predictions.

The Ninth Annual Conference on Large Hadron Collider Physics - LHCP2021

7-12 June 2021

Online 


\section{Introduction}

At hadron colliders, top quarks are produced predominantly in pairs via the flavor-conserving strong interaction. Alternatively, top quarks can be also produced singly through the electroweak interaction. The large amount of LHC data recorded by the ATLAS [1] and CMS [2] experiments up to date allows probing very rare Standard Model (SM) processes as the associated top quark production processes presented in this document. These processes become fully accessible with Run $2 \mathrm{LHC}$ data at $13 \mathrm{TeV}$ despite their small cross sections and are important background contributions for measurements like $\mathrm{t} \overline{\mathrm{t}} \mathrm{H}$. They are among the most massive signatures produced at the LHC. Finally, these processes receive a significant enhancement in beyond standard models. The following

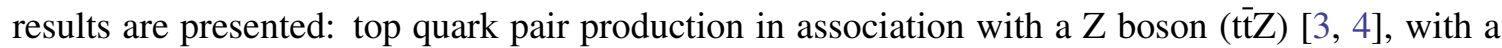
photon $(\mathrm{t} \overline{\mathrm{t}} \gamma)[5,6]$ and single top quark production in association with a $\mathrm{Z}$ boson (tZq) $[7,8]$ by CMS and ATLAS, respectively.

\section{Top quark pair production in association with a $\mathrm{Z}$ boson}

The $t \bar{t} Z$ production is the most sensitive process for the coupling of the top quark to the $\mathrm{Z}$ boson. Measurements of both the inclusive and differential production cross sections of tt'Z are presented. The measurements are performed by targeting final states with three or four isolated leptons (electrons or muons). ATLAS uses the full Run 2 dataset with an integrated luminosity of $139 \mathrm{fb}^{-1}$ recorded from 2015 to 2018 . The data sample used by CMS corresponds to an integrated luminosity of $77.5 \mathrm{fb}^{-1}$ collected during 2016 and 2017.

The inclusive cross section measured by ATLAS is $0.99 \pm 0.05$ (stat) \pm 0.08 (syst) pb, in agreement with the most precise theoretical predictions. The differential measurements are presented as a function of a number of kinematic variables which probe the kinematics of the $t \bar{t} Z$ system as shown in Fig. 1 (left). Both absolute and normalised differential cross-section measurements are performed at particle and parton levels for specific fiducial volumes and are compared with theoretical predictions at different levels of precision. Overall, good agreement is observed between the unfolded data and the predictions. The production cross section measured by CMS is $0.95 \pm 0.05$ (stat) \pm 0.06 (syst) pb, also in good agreement with SM predictions. Differential cross sections are measured as functions of the angular distribution of the negatively charged lepton from the $\mathrm{Z}$ boson decay and the transverse momentum of the $\mathrm{Z}$ boson as shown in Fig. 1 (right).

\section{Top quark pair production in association with a photon}

The production cross section of a top quark pair in association with a photon is measured by ATLAS and CMS based on a data set recorded during the full LHC Run 2. The ATLAS measurements are performed in a fiducial volume defined at parton level. Events with exactly one photon, one electron and one muon of opposite sign, and at least two jets, of which at least one is b-tagged, are selected. The fiducial cross-section is measured to be $39.6_{-2.3}^{+2.7} \mathrm{fb}$. Differential cross-sections as functions of several observables are compared with state-of-the-art Monte Carlo simulations and next-to-leading-order theoretical calculations.

The CMS measurement is performed in events with a well isolated, highly energetic lepton (electron or muon), with at least three jets from the hadronization of quarks and one isolated 


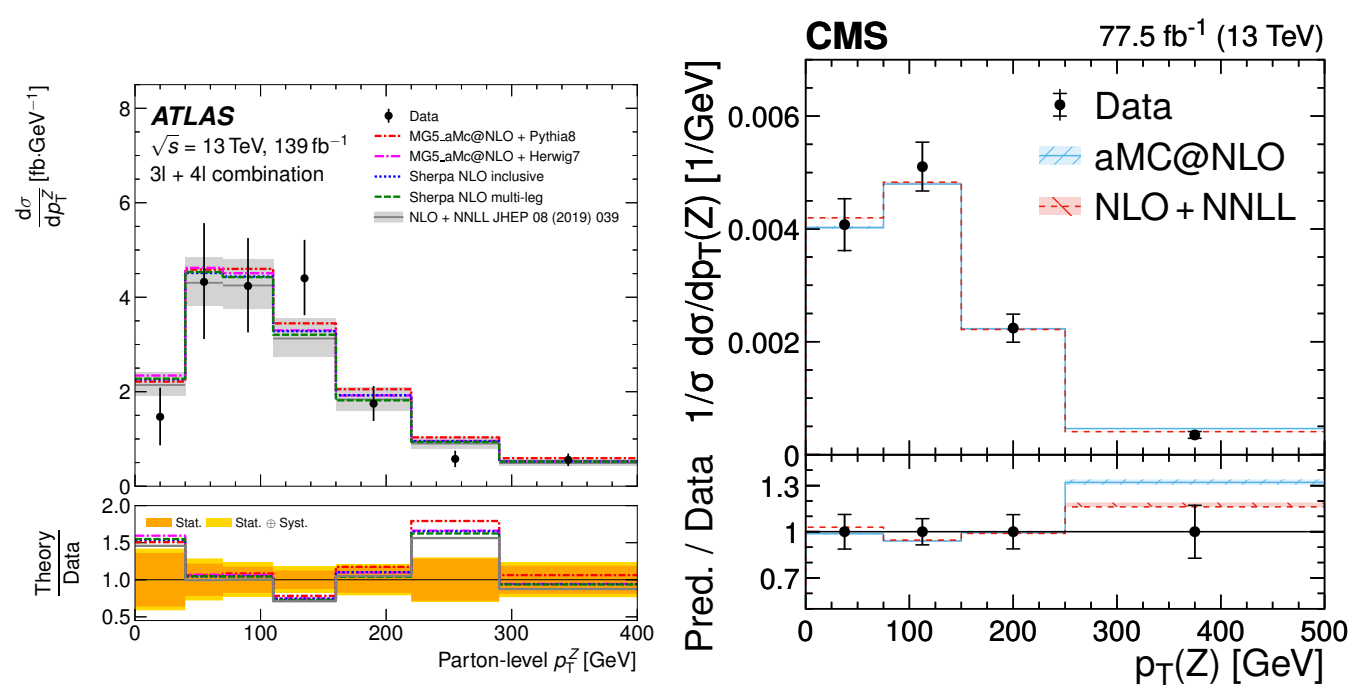

Figure 1: Left: Absolute differential t⿱tZZ cross sections measured by ATLAS at parton level as a function of the transverse momentum of the reconstructed $\mathrm{Z}$ boson [4]. Right: CMS measured differential tīZ production cross sections in the full phase space as a function of the transverse momentum of the $\mathrm{Z}$ boson. Shown are the normalized cross sections [3]. The data are represented by the points. The inner (outer) vertical lines indicate the statistical (total) uncertainties. The solid histogram shows the prediction from the MadGraph 5_aMC@NLO MC simulation, and the dashed histogram shows the theory prediction at NLO+NNLL accuracy. The hatched bands indicate the theoretical uncertainties in the predictions, as defined in the text. The lower panel displays the ratios of the predictions to the measurement.

photon. The analysis makes use of simultaneous likelihood fits in several signal and control regions to distinguish the $\bar{t} \gamma$ signal process from various backgrounds. The inclusive cross section for a photon with transverse momentum of $\mathrm{p}_{\mathrm{T}} \geq 20 \mathrm{GeV}$ is measured as $800 \pm 46$ (syst) \pm 7 (stat) fb, in good agreement with the prediction from the SM. These preliminary results are superseded in Ref. [9], submitted to JHEP. The measurement is also carried out differentially in several kinematic observables and interpreted in the framework of the SM effective field theory. All measurements are in agreement with the predictions from the SM.

\section{Summary of $\mathrm{tt} X$ measurements}

The ATLAS and CMS measurements of $\overline{t t X}(\mathrm{X}=\mathrm{W}, \mathrm{Z}$ or $\gamma)$ cross sections at $13 \mathrm{TeV}$ are summarized in Fig. 2. The tti W and tt' $Z$ cross section measurements are compared to the NLO QCD and EW theoretical calculation complemented with NNLL resummation, while the $\mathrm{t} \overline{\mathrm{t}} \gamma$ cross section measurement is compared to the NLO QCD theoretical calculation. The "Vis 1" and "Vis 2" labels in the figure highlight that the relevant phase space used for the ATLAS and CMS t⿱t$\gamma$ measurements are different. The theory prediction and experimental result of the ATLAS measurement $\overline{\mathrm{t}} \gamma+\mathrm{tW} \gamma$ process is multiplied by a factor 20 to allow for easy visualization on the same scale. The theory band represents uncertainties due to renormalisation and factorisation scales and parton density functions. Complementary theory predictions for the titW and $\bar{t} \bar{Z}$ processes are also available in [11]. 


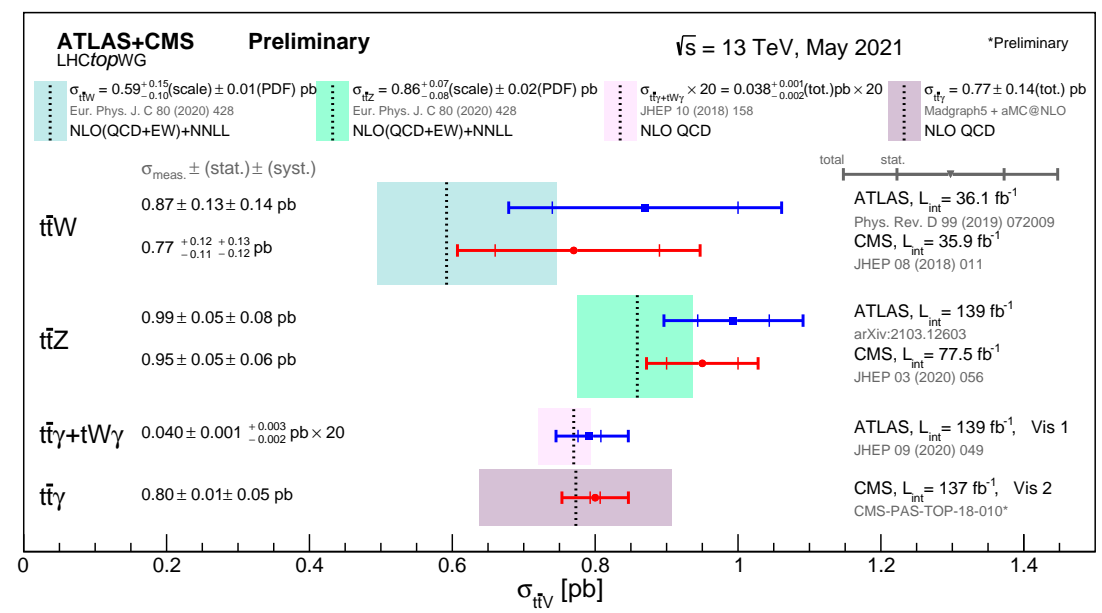

Figure 2: Summary of ATLAS and CMS measurements of $\overline{\mathrm{t}} \mathrm{X}(\mathrm{X}=\mathrm{W}, \mathrm{Z}$ or $\gamma)$ cross sections at $13 \mathrm{TeV}$ [10].

\section{Single top quark production in association with a $\mathrm{Z}$ boson}

Single top quark production in association with a $\mathrm{Z}$ boson, where the $\mathrm{Z}$ boson decays to a pair of charged leptons, is measured in the trilepton channel by ATLAS and CMS based on a data set recorded during the full LHC Run 2. Events containing three isolated charged leptons (electrons or muons) are selected. The main backgrounds are from t' $\overline{\mathrm{t}} \mathrm{Z}$ and diboson production. ATLAS uses neural networks to improve the background rejection and extract the signal. The measured cross-section including non-resonant dilepton pairs with $\mathrm{m}_{\mathrm{l}^{+} \mathrm{l}^{-}}>30 \mathrm{GeV}$ is $97 \pm 13$ (stat) \pm 7 (syst) fb, consistent with the SM prediction. CMS measures $87.9_{-7.3}^{+7.5}$ (stat) ${ }_{-6.0}^{+7.3}$ (syst) fb also including non-resonant dilepton pairs.

For the first time, the inclusive $\mathrm{tZq}$ cross sections are also measured by CMS separately for top quark and antiquark production, obtaining 62.2 $2_{-5.7}^{+5.9}$ (stat) ${ }_{-3.7}^{+4.4}$ (syst) fb and 26.1 $1_{-4.6}^{+4.8}$ (stat) ${ }_{-2.8}^{+3.0}$ (syst) fb, respectively. The differential tZq cross sections are measured for the first time at parton and particle levels using a binned maximum likelihood-based unfolding. The results are mostly compatible with the SM predictions using both the four- and five-flavor schemes. From the differential distribution of the top quark polarization angle, the top quark spin asymmetry is measured to be $0.58_{-0.16}^{+0.15}$ (stat) \pm 0.06 (syst), in agreement with the SM prediction.

\section{References}

[1] ATLAS Collaboration, The ATLAS Experiment at the CERN LHC, JINST 3 S8003 (2008).

[2] CMS Collaboration, The CMS Experiment at the CERN LHC, JINST 3 S8004 (2008).

[3] CMS Collaboration, Measurement of top quark pair production in association with a $Z$ boson in proton-proton collisions at $\sqrt{s}=13 \mathrm{TeV}$, JHEP 03 (2020) 056 [arXiv:1907.11270]. 
[4] ATLAS Collaboration, Measurements of the inclusive and differential production cross sections of a top-quark-antiquark pair in association with a $Z$ boson at $\sqrt{s}=13 \mathrm{TeV}$ with the ATLAS detector, EPJC 81 (2021) 737, [arXiv:103.12603].

[5] CMS Collaboration,Measurement of the inclusive and differential $\mathrm{t} \overline{\mathrm{t}}+\gamma$ cross section and EFT interpretation in the single lepton channel at $\sqrt{s}=13 \mathrm{TeV}$,CMS-PAS-TOP-18-010.

[6] ATLAS Collaboration, Measurements of inclusive and differential cross-sections of combined $t \bar{t} \gamma$ and $t W \gamma$ production in the e $\mu$ channel at $13 \mathrm{TeV}$ with the ATLAS detector, JHEP 09 (2020) 049, [arXiv:2007.06946"].

[7] CMS Collaboration, Inclusive and differential cross section measurements of single top quark production in association with a $Z$ boson in proton-proton collisions at $\sqrt{s}=13 \mathrm{TeV}$, CMSPAS-TOP-20-010.

[8] ATLAS Collaboration,Observation of the associated production of a top quark and a $Z$ boson in pp collisions at $\sqrt{s}=13 \mathrm{TeV}$ with the ATLAS detector, JHEP 07 (2020) 124 [arXiv:2002.07546],

[9] CMS Collaboration,Measurement of the inclusive and differential tit $\gamma$ cross sections in the single-lepton channel and EFT interpretation at $\sqrt{s}=13 \mathrm{TeV}$,[arXiv:2107.01508].

[10] LHC TOP Working Group, https://twiki.cern.ch/twiki/bin/view/LHCPhysics/LHCTopWGSummaryPlots

[11] A. Broggio et al., JHEP 08 (2019) 039 [arXiv:1610.07922]. 